\title{
Science and Medicine
}

\author{
Ze'ev Hochberg
}

\section{New paradigms: personalized epigenome}

\section{Personalized epigenomic signatures that are stable over time and covary with body mass index}

Feinberg AP, Irizarry RA, Fradin D, Aryee MJ, Murakami P, Aspelund T, Eiriksdottir G, Harris TB, Launer L, Gudnason V, Fallin MD

Center for Epigenetics, Johns Hopkins School of Medicine, Baltimore, Md., USA.

afeinberg@jhu.edu

Sci Transl Med 2010;2:49ra67.

Background: The epigenome consists of non-sequence-based modifications, such as DNA methylation, that are heritable during cell division and that may affect normal phenotypes and predisposition to disease.

Methods: This study performed an unbiased genome-scale analysis of $\sim 4$ million CpG sites in 74 individuals with comprehensive array-based relative methylation (CHARM) analysis.

Results: They found 227 regions that showed extreme interindividual variability (variably methylated regions (VMRs)) across the genome, which are enriched for developmental genes based on Gene Ontology analysis. Furthermore, half of these VMRs were stable within individuals over an average of 11 years, and these VMRs defined a personalized epigenomic signature. Four of these VMRs showed covariation with body mass index consistently at two study visits and were located in or near genes previously implicated in regulating body weight or diabetes.

We tend to segregate diseases by the environment (such as diet) and genetics, but it is the combination of both that counts in each case. Environmental cues speak the language of epigenetics as they interact with genes. This work offers a strategy for epigenetic-based approach to identifying patients at risk of common disease. A survey of millions of epigenetic modifications has uncovered a handful associated with body mass index. To build large-scale maps of 'epigenomes', the Feinberg's lab examined 4.5 million places along the human genome where methylation can occur. They report 227 DNA regions with different methylation patterns from person to person. Of those, 119 remained the same over the decade within each person, providing an epigenetic fingerprint for each individual. Six such methylation sites harbored genes that have previously been linked to or were suspected of contributing to obesity or diabetes. How we use this new personalized epigenome is yet to be seen. 


\title{
Dnmt3a-dependent nonpromoter DNA methylation facilitates transcription of neurogenic genes
}

\author{
Wu H, Coskun V, Tao J, Xie W, Ge W, Yoshikawa K, Li E, Zhang Y, Sun YE \\ Department of Molecular and Medical Pharmacology, University of California Los Angeles (UCLA), Los Angeles, \\ Calif., USA. \\ haowu7@gmail.com
}

Science 2010;329:444-8.

Background: DNA methylation at proximal promoters facilitates lineage restriction by silencing cell typespecific genes. However, euchromatic DNA methylation frequently occurs in regions outside promoters. The functions of such nonproximal promoter DNA methylation are unclear.

Results: This paper shows that the de novo DNA methyltransferase Dnmt3a is expressed in postnatal neural stem cells (NSCs) and is required for neurogenesis. Genome-wide analysis of postnatal NSCs indicates that Dnmt3a occupies and methylates intergenic regions and gene bodies flanking proximal promoters of a large cohort of transcriptionally permissive genes, many of which encode regulators of neurogenesis. Surprisingly, Dnmt3a-dependent nonproximal promoter methylation promotes expression of these neurogenic genes by functionally antagonizing polycomb repression.

Conclusion: Nonpromoter DNA methylation by Dnmt3a may be used for maintaining active chromatin states of genes critical for development.

The methyltransferases (Dnmts) modify mammalian genomes by cytosine methylation, an epigenetic mark that is essential for normal development and primarily occurs at CpG dinucleotides. Most CpGrich regions ( $\mathrm{CpG}$ islands) overlap with proximal promoters, where DNA methylation is linked to gene silencing. Yet, GWAS have shown that DNA methylation is widespread and predominantly takes place in regions outside proximal promoters. This article deals with Dnmt3a, which is not required for maintaining methylation at heterochromatic repeat regions, and Dnmt3a-null mice appear to be grossly normal at birth, but acquire developmental defects postnatally and die. This study mapped genome-wide Dnmt3a occupancy in postnatal neural stem cells, and identified 30,417 Dnmt3a binding sites, many of which are nonproximal promoters and encode regulators of neurogenesis. The horizon for nonpromoter DNA methylation by Dnmt3a may include more genes in more organs that are critical for development.

\section{Cousins are for life}

\section{A draft sequence of the Neandertal genome}

Green RE, Krause J, Briggs AW, Maricic T, Stenzel U, Kircher M, Patterson N, Li H, Zhai W, Fritz MH, Hansen NF, Durand EY, Malaspinas AS, Jensen JD, Marques-Bonet T, Alkan C, Prufer K, Meyer M, Burbano HA, Good JM, Schultz R, Aximu-Petri A, Butthof A, Hober B, Hoffner B, Siegemund M, Weihmann A, Nusbaum C, Lander ES, Russ C, Novod N, Affourtit J, Egholm M, Verna C, Rudan P, Brajkovic D, Kucan Z, Gusic I, Doronichev VB, Golovanova LV, Lalueza-Fox C, de la Rasilla M, Fortea J, Rosas A, Schmitz RW, Johnson PL, Eichler EE, Falush D, Birney E, Mullikin JC, Slatkin M, Nielsen R, Kelso J, Lachmann M, Reich D, Paabo S

Department of Evolutionary Genetics, Max Planck Institute for Evolutionary Anthropology, Leipzig, Germany. green@ eva.mpg.de

Science 2010;328:710-22.

Background: Neanderthals, the closest evolutionary relatives of present-day humans, lived in large parts of Europe and western Asia before disappearing 30,000 years ago. This paper presents a draft sequence of the Neanderthal genome composed of more than 4 billion nucleotides from 3 individuals.

Results: Comparisons of the Neanderthal genome to the genomes of 5 present-day humans from different parts of the world identify a number of genomic regions that may have been affected by positive 
selection in ancestral modern humans, including genes involved in metabolism and in cognitive and skeletal development. They show that Neanderthals shared more genetic variants with present-day humans in Eurasia than with present-day humans in sub-Saharan Africa, suggesting that gene flow from Neanderthals into the ancestors of non-Africans occurred before the divergence of Eurasian groups from each other.

This draft of the Neanderthal genome is a composite sequence from 3 females who lived in Croatia 38,000-44,000 years ago and reveals the evolution of these extinct hominids and us: modern Europeans and Asians - but not Africans - have inherited between 1 and $4 \%$ of their genes from Neanderthals. Apparently, Neanderthals interbred with modern humans after they left Africa at least 80,000 years ago but before they spread into Europe and Asia. This concept challenges a model suggesting that modern humans completely replaced archaic hominids such as Neanderthals. The catalog includes 78 differences from modern humans in genes that encode proteins that are important for cognitive (when mutated in humans, some of these genes contribute to diseases such as schizophrenia, Down syndrome, and autism) and skeletal development, wound healing, the beating of sperm flagella, and gene transcription for proteins expressed in the skin (including skin color), sweat glands, hair roots, and adaptive genes to new climates and environments as modern humans spread around the globe.

\section{Amazing: creation is continuous}

\section{Creation of a bacterial cell controlled by a chemically synthesized genome}

Gibson DG, Glass J, Lartigue C, Noskov VN, Chuang RY, Algire MA, Benders GA, Montague MG, Ma L, Moodie MM, Merryman C, Vashee S, Krishnakumar R, Assad-Garcia N, Andrews-Pfannkoch C, Denisova EA, Young L, Qi ZQ, Segall-Shapiro TH, Calvey CH, Parmar PP, Hutchison CA 3rd, Smith HO, Venter JC The J. Craig Venter Institute, Rockville, Md., USA.

Science 2010;329:52-6.

This paper reports the design, synthesis, and assembly of the 1.08-mega-basepair Mycoplasma mycoides JCVI-syn 1.0 genome starting from digitized genome sequence information and its transplantation into a M. capricolum recipient cell to create new M. mycoides cells that are controlled only by the synthetic chromosome. The only DNA in the cells is the designed synthetic DNA sequence, including 'watermark' sequences and other designed gene deletions and polymorphisms, and mutations acquired during the building process. The new cells have expected phenotypic properties and are capable of continuous self-replication.

Craig Venter and his team had created an entirely synthetic genome that generated a viable and replicating cell. Aiming at a bacterium with one of the smallest genomes - Mycoplasma genitalium - the team predicted that of its 480 protein-coding genes, only 300 or so were essential. They then insert it into a genome-less cell and demonstrate that it could initiate and continue cell division. The ethical consequences are problematic, to say the least. The team was aware of this and claim that their ultimate goal is to carve away at the synthetic genome and repeat transplantation experiments until no more genes can be disrupted and the genome is as small as possible. This will help understand the function of every gene in a cell and what DNA is required to sustain life in its simplest form. 


\section{A liver-derived secretory protein, selenoprotein $\mathbf{P}$, causes insulin resistance}

Misu H, Takamura T, Takayama H, Hayashi H, Matsuzawa-Nagata N, Kurita S, Ishikura K, Ando H, Takeshita Y, Ota T, Sakurai M, Yamashita T, Mizukoshi E, Honda M, Miyamoto K, Kubota T, Kubota N, Kadowaki T, Kim HJ, Lee IK, Minokoshi Y, Saito Y, Takahashi K, Yamada Y, Takakura N, Kaneko S

Department of Disease Control and Homeostasis, Kanazawa University Graduate School of Medical Science, Ishikawa, Japan.

Cell Metab 2010;12:483-95.

Background: The liver may regulate glucose homeostasis by modulating the sensitivity/resistance of peripheral tissues to insulin, by way of the production of secretory proteins, termed hepatokines.

Results: This paper demonstrates that selenoprotein $\mathrm{P}(\mathrm{SeP})$, a liver-derived secretory protein, causes insulin resistance. Using serial analysis of gene expression (SAGE) and DNA chip methods, they found that hepatic SeP mRNA levels correlated with insulin resistance in humans. Administration of purified SeP impaired insulin signaling and dysregulated glucose metabolism in both hepatocytes and myocytes. Conversely, both genetic deletion and RNA interference-mediated knockdown of SeP improved systemic insulin sensitivity and glucose tolerance in mice. The metabolic actions of SeP were mediated, at least partly, by inactivation of adenosine monophosphate-activated protein kinase (AMPK).

Conclusions: These results demonstrate a role of $\mathrm{SeP}$ in the regulation of glucose metabolism and insulin sensitivity, and suggest that $\mathrm{SeP}$ may be a therapeutic target for type 2 diabetes.

For pediatric endocrinologists the liver always generated hormones such as IGFs. Now, the liver secretes a hormone that participates in the pathogenesis of insulin resistance, which the authors refer to as a 'hepatokine'. Liver expression and blood levels of selenoprotein P (SeP) are higher in people with diabetes compared to healthy people. When the researchers gave normal mice SeP, they became insulin-resistant and their blood sugar levels rose. Blocking SeP activity in the livers of diabetic and obese mice improved their sensitivity to insulin and lowered blood sugar levels. In the previous decade we learnt that fat tissue is a main contributor to the development of insulin resistance by producing fat adipokines. Apparently SeP and adipokine cooperate, and eventually other hepatokines will have roles in the body.

\section{Food for thought: old dog new tricks}

\section{Cancer: an old disease, a new disease or something in between?}

David AR, Zimmerman MR

KNH Centre of Biomedical Egyptology, The University of Manchester, Manchester, UK.

rosalie.david@manchester.ac.uk

Nat Rev Cancer 2010;10:728-33.

In industrialized societies, cancer is second only to cardiovascular disease as a cause of death. The history of this disorder has the potential to improve our understanding of disease prevention, etiology, pathogenesis and treatment. A striking rarity of malignancies in ancient physical remains might indicate that cancer was rare in antiquity, and so poses questions about the role of carcinogenic environmental factors in modern societies. Although the rarity of cancer in antiquity remains undisputed, the first published histological diagnosis of cancer in an Egyptian mummy demonstrates that new evidence is still forthcoming.

Investigation of hundreds of Egyptian mummies showed that cancer was extremely rare in antiquity. Whereas this might be due to people living now longer, the case of childhood cancer proves that the rise is not simply due to people living longer. Yet, we know of nothing in the natural environment that can cause cancer. Hence cancer must be due to a mismatch between our genes and man- 
made environment, such as pollution, our diet and lifestyle. The virtual absence of malignancies in mummies must be interpreted as indicating their infrequency in antiquity. In the same token, evidence of cancer in animal fossils, non-human primates and early humans is scarce.

\section{New mechanism: bone of contention}

\section{Insulin receptor signaling in osteoblasts regulates postnatal bone acquisition and body composition}

Fulzele K, Riddle RC, DiGirolamo DJ, Cao X, Wan C, Chen D, Faugere MC, Aja S, Hussain MA, Bruning JC, Clemens TL

Department of Orthopedic Surgery, Johns Hopkins University School of Medicine, Baltimore, Md., USA.

Cell 2010;142:309-19.

Background: Global energy balance in mammals is controlled by the actions of circulating hormones that coordinate fuel production and utilization in metabolically active tissues. Bone-derived osteocalcin, in its undercarboxylated, hormonal form, regulates fat deposition and is a potent insulin secretagogue.

Methods and Results: Here, they show that insulin receptor (IR) signaling in osteoblasts controls osteoblast development and osteocalcin expression by suppressing the Runx 2 inhibitor Twist 2 . Mice lacking IR in osteoblasts have low circulating undercarboxylated osteocalcin and reduced bone acquisition due to decreased bone formation and deficient numbers of osteoblasts. With age, these mice develop marked peripheral adiposity and hyperglycemia accompanied by severe glucose intolerance and insulin resistance. The metabolic abnormalities in these mice are improved by infusion of undercarboxylated osteocalcin.

Conclusions: The existence of a bone-pancreas endocrine loop, through which insulin signaling in the osteoblast ensures osteoblast differentiation and stimulates osteocalcin production, in turn regulates insulin sensitivity and pancreatic insulin secretion.

Here is a new target tissue for insulin; insulin signaling in osteoblasts is required for normal bone acquisition and stimulation of osteoblast production. The latter generates osteocalcin, which turns out to be an insulin secretagogue. Mice lacking insulin receptors in osteoblasts develop peripheral insulin resistance, which is ameliorated by osteocalcin infusion as shown in mutant insulin receptor mice.

\section{Concepts revised: losing one's best pounds}

\section{Weight loss and lipolysis promote a dynamic immune response in murine adipose tissue}

Kosteli A, Sugaru E, Haemmerle G, Martin JF, Lei J, Zechner R, Ferrante AW, Jr.

Department of Medicine, Naomi Berrie Diabetes Center, Columbia University, New York, N.Y., USA.

J Clin Invest 2010;120:3466-79.

Background: Obesity elicits an immune response characterized by myeloid cell recruitment to key metabolic organs, including adipose tissue. However, the response of immune cells to nonpathologic metabolic stimuli has been less well studied, and the factors that regulate the metabolic-dependent accumulation of immune cells are incompletely understood.

Methods: This study characterized the response of adipose tissue macrophages (ATMs) to weight loss and fasting in mice and identified a role for lipolysis in ATM recruitment and accumulation.

Results: They found that the immune response to weight loss was dynamic; caloric restriction of high-fat diet-fed mice led to an initial increase in ATM recruitment, whereas ATM content decreased following 
an extended period of weight loss. The peak in ATM number coincided with the peak in the circulating concentrations of FFA and adipose tissue lipolysis, suggesting that lipolysis drives ATM accumulation. Indeed, fasting or pharmacologically induced lipolysis rapidly increased ATM accumulation, adipose tissue chemoattractant activity, and lipid uptake by ATMs. Conversely, dietary and genetic manipulations that reduced lipolysis decreased ATM accumulation. Depletion of macrophages in adipose tissue cultures increased expression of adipose triglyceride lipase and genes regulated by FFA, and increased lipolysis.

Conclusions: These data suggest that local lipid fluxes are central regulators of ATM recruitment and that once recruited, ATMs form lipid-laden macrophages that can buffer local increases in lipid concentration.

Macrophages in the adipose tissue of obese individuals contribute to insulin resistance. This study now shows that lipolysis plays a key role in recruiting macrophages to adipose tissue. Soon after weight loss or fasting in mice, macrophages in fat paradoxically increase, accumulate lipid and dampen lipolysis. The concept that macrophages buffer lipid accumulation in adipose tissue is reminiscent of their role in atherosclerosis, where an adaptive role of macrophages in clearing lipid from the vessel wall becomes maladaptive under high lipid burden. So, similar to the role of macrophages in controlling local excess cholesterol in the arteries, macrophages can respond to acute changes in lipolysis and migrate to adipose tissues, where they phagocytose excess lipids without causing inflammation. As triglyceride stores are depleted and basal lipolysis decreases during ongoing weight loss, macrophages leave the adipose tissue and metabolic function is protected.

\section{On clinical trial and their reports (two articles)}

\section{Outcome reporting among drug trials registered in ClinicalTrials.gov}

Bourgeois FT, Murthy S, Mandl KD

Division of Emergency Medicine, Children's Hospital Boston, Boston, Mass., USA.florence.bourgeois@childrens. harvard.edu

Ann Intern Med 2010;153:158-66.

Background: Clinical trial registries are in widespread use to promote transparency around trials and their results. This paper describes characteristics of drug trials listed in ClinicalTrials.gov and examine whether the funding source of these trials is associated with favorable published outcomes

Methods: An observational study of safety and efficacy trials for anticholesteremics, antidepressants, antipsychotics, proton-pump inhibitors, and vasodilators conducted between 2000 and 2006 . Publications resulting from the trials for the 5 drug categories of interest were identified, and data were abstracted on the trial record and publication, including timing of registration, elements of the study design, funding source, publication date, and study outcomes. Assessments were based on the primary funding categories of industry, government agencies, and nonprofit or nonfederal organizations.

Results: Among 546 drug trials, 346 (63\%) were primarily funded by industry, 74 (14\%) by government sources, and $126(23 \%)$ by nonprofit or nonfederal organizations. Trials funded by industry were more likely to be phase 3 or 4 trials $(88.7 \%$; $<<0.001$ across groups), to use an active comparator in controlled trials $(36.8 \% ; \mathrm{p}=0.010$ across groups), to be multicenter $(89.0 \% ; \mathrm{p}<0.001$ across groups), and to enroll more participants (median sample size, 306 participants; $\mathrm{p}<0.001$ across groups). Overall, $362(66.3 \%)$ trials had published results. Industry-funded trials reported positive outcomes in $85.4 \%$ of publications, compared with $50.0 \%$ for government-funded trials and $71.9 \%$ for nonprofit or nonfederal organization-funded trials $(\mathrm{p}<0.001)$. Trials funded by nonprofit or nonfederal sources with industry contributions were also more likely to report positive outcomes than those without industry funding (85.0 vs. $61.2 \%$; $=0.013$ ). Rates of trial publication within 24 months of study completion ranged from $32.4 \%$ among industry-funded trials to $56.2 \%$ among nonprofit or nonfederal organization-funded trials without industry contributions ( $\mathrm{p}=0.005$ across groups). 
Conclusion: In this sample of registered drug trials, those funded by industry were less likely to be published within 2 years of study completion and were more likely to report positive outcomes than were trials funded by other sources.

\section{Frequency and reasons for outcome reporting bias in clinical trials: interviews with trialists}

Smyth RM, Kirkham JJ, Jacoby A, Altman DG, Gamble C, Williamson PR

Centre for Medical Statistics and Health Evaluation, University of Liverpool, Liverpool, UK.

BMJ 2011;342:C7153.

Background: This report attempts to provide information on the frequency and reasons for outcome reporting bias in clinical trials.

Methods: Trial protocols were compared with subsequent publication(s) to identify any discrepancies in the outcomes reported, and telephone interviews were conducted with the respective trialists to investigate more extensively the reporting of the research and the issue of unreported outcomes. Chief investigators, or lead or co-authors of trials, were identified from two sources: trials published since 2002 covered in Cochrane systematic reviews where at least one trial analyzed was suspected of being at risk of outcome reporting bias (issue 4, 2006; issue 1, 2007, and issue 2, 2007 of the Cochrane Library), and a random sample of trial reports indexed on PubMed between August 2007 and July 2008.

Main Outcome Measures: Frequency of incomplete outcome reporting - signified by outcomes that were specified in a trial's protocol but not fully reported in subsequent publications - and trialists' reasons for incomplete reporting of outcomes.

Results: 268 trials were identified for inclusion (183 from the cohort of Cochrane systematic reviews and 85 from PubMed). Initially, 161 respective investigators responded to our requests for interview, 130 $(81 \%)$ of whom agreed to be interviewed. However, failure to achieve subsequent contact, obtain a copy of the study protocol, or both meant that final interviews were conducted with $59(37 \%)$ of the 161 trialists. Sixteen trial investigators failed to report analyzed outcomes at the time of the primary publication, 17 trialists collected outcome data that were subsequently not analyzed, and 5 trialists did not measure a prespecified outcome over the course of the trial. In almost all trials in which prespecified outcomes had been analyzed but not reported $(15 / 16,94 \%)$, this underreporting resulted in bias. In nearly a quarter of trials in which prespecified outcomes had been measured but not analyzed (4/17, $24 \%$ ), the 'direction' of the main findings influenced the investigators' decision not to analyze the remaining data collected. In $14(67 \%)$ of the 21 randomly selected PubMed trials, there was at least one unreported efficacy or harm outcome. More than a quarter $(6 / 21,29 \%)$ of these trials were found to have displayed outcome reporting bias.

Conclusions: The prevalence of incomplete outcome reporting is high. Trialists seemed generally unaware of the implications for the evidence base of not reporting all outcomes and protocol changes. A general lack of consensus regarding the choice of outcomes in particular clinical settings was evident and affects trial design, conduct, analysis, and reporting.

Selective reporting and data suppression from clinical trials are well documented, leading to all of us managing patients on the basis of an incomplete and biased trial results. The requirement to register clinical trials ahead of starting has improved it to some extent, but the raw dataset and the protocol approved by the research ethics committee usually remain elusive. These two reports conclude with a proposal that journals should require submission of detailed dataset and the full protocols. 